\title{
Evaluation of Protracted Cisplatinum Infusion in Advanced Anaplastic Thyroid Cancer
}

\author{
Ghosh $\mathbf{G}^{1}$, Singh $\mathbf{O P}^{2}$ \\ ${ }^{1}$ Dr. Gopa Ghosh, Consultant, Radiation \& Clinical Oncology, L N Medical College \& Research Centre, Bhopal, India. ${ }^{2}$ Dr. \\ O.P. Singh, Professor, Radiation \& Clinical Oncology, Gandhi Medical College, Bhopal, India.
}

Address for correspondence: Dr Gopa Ghosh, Email: gopaghosh571@yahoo.in.

\begin{abstract}
Introduction: Anaplastic thyroid cancer (ATC) constitutes 1-3\% of all thyroid malignancies. Most of the patients of anaplastic thyroid cancer presents with advanced inoperable lesion associated with neck mass dysphagia, and SVC syndrome. At this stage only treatment that can be offered is chemotherapy. Present study evaluates protracted ( 8 hr) Cisplatinum (CDDP) infusion along with doxorubucin in comparison to conventional (1hr) Cisplatinum + doxorubucin in advanced anaplastic thyroid cancer in terms of tolerance, toxicities and response rates. Methods: 32 previously untreated cases of stage III/IV inoperable anaplastic thyroid cancer were included in the present study. Patients were divided into 2 arms of 16 patients each. Arm I (16 patients) received $1 \mathrm{hr}$ Cisplatin (CDDP) infusion 75mg/m2 + Doxorubicin 60mg/m2 infusion \& Arm II (16 patients) received protracted $8 \mathrm{hrs}$ CDDP infusion $75 \mathrm{mg} / \mathrm{m} 2+$ Doxorubicin $60 \mathrm{mg} / \mathrm{m} 2$ infusion. All patients were evaluated for tolerance, toxicities and response rate. Results: Arm II patients showed better locoregional response with CR 31.25\%, PR 50\%, SD 6.25\%,PrD. $12.5 \%$ as compared to Arm I patients with CR 12.5\%, PR 43.75\%, SD 18.75\%, Pr. D 25\%, p=.02. Toxicities like mucositis, nausea \& vomiting, diarrhea, nephrotoxicity were also significantly less in Arm II. After completion of 3-4cycles of Induction CT, all patients were treated with External Radiotherapy of 60-66Gy, followed with 2-3 cycles of adjuvant CT. Conclusion: From the present study it can be concluded that protracted cisplatinum infusion along with other chemotherapeutic drugs is an effective and acceptable CT regimen in advanced inoperable thyroid cancer.
\end{abstract}

Key words: Anaplastic thyroid cancer, chemotherapy, prolonged cisplatinum

\section{Introduction}

Historically, ATC was said to constitute $5-15 \%$ of all thyroid carcinomas in UnitedStates ${ }^{1}$ and $10-50 \%$ in Europe $^{2}$. Current epidemiologic studies indicate that this lethal form of thyroid cancer has decreased to between 1-

Manuscript received: $13^{\text {th }}$ Aug 2013

Reviewed: $26^{\text {th }}$ Aug 2013

Author Corrected: $29^{\text {th }}$ Aug 2013

Accepted for Publication: $20^{\text {th }}$ Sep 2013
$3 \%$ worldwide of the total number of $\operatorname{cases}^{3}$. The decrease over time may be partially related to iodine prophylaxis and an overall decrease in endemic iodine deficient goiter ${ }^{3-6}$. Anaplastic cancer originates from follicular cells of thyroid. Tumor grows rapidly and prognosis is grave. Local invasion of structures like trachea, esophagus and superior mediastinum is followed by distant metastasis \& 
death $^{4-7}$. Management of anaplastic thyroid cancer advocates debulking by surgical resection, External radiotherapy $(\mathrm{RT})$ chemotherapy $(\mathrm{CT})^{7-10}$.

Most of the patients present with advanced non operable lesion associated with neck mass, dysphagia, dysphonia and SVC syndrome ${ }^{6,7}$. In such cases the only form of treatment that can be offered is chemotherapy. These cancers do not concentrate iodine ${ }^{6}$.

The most common chemotherapeutic drug used in such cases is Doxorubicin used singly or in combination. Combination of Cisplatinum and Doxorubicin is the most common combination that has been widely tried ${ }^{7,10-12}$.

Cisplatinum is usually administered as $1 \mathrm{hr}$ infusion but nowadays-protracted infusion like $6 \mathrm{hrs}$ or $24 \mathrm{hrs}$ has been tried in advanced head\& neck cancer ${ }^{11-13}$. Other chemotherapeutic regimens that have been tried are Adriamycin, Bleomycin, and Paclitaxel ${ }^{7,14}$.

Combinations like CDDP +Doxorubicin +Etoposide+Peplomycin have been investigated by some researchers like (Japanese society of thyroid surgery) ${ }^{15}$.

Voig et $\mathrm{al}^{16}$ evaluated Gemcitabine +CDDP in anaplastic thyroid cancer. Protracted cisplatinum infusion has been explored in Paediatric tumours like neuroblastoma as well ${ }^{17}$.

In view of prolonged cisplatinum infusion being tried in Head \& Neck cancer previously as mentioned above, protracted $8 \mathrm{hrs}$ cisplatinum infusion in combination with Doxorubicin has been evaluated in advanced anaplastic thyroid carcinoma in terms of response rate and toxicity as compared to $1 \mathrm{hr}$ infusion in this study

\section{Material and Methods}

32 previously untreated histopathologically proved advanced thyroid carcinoma patients who were reported in department of Radiotherapy S.S. Medical College Rewa \& Gandhi Medical College Bhopal between Jan 2004 to Jan 2009 constitutes the study subject.
Inclusion criteria of patients were:

1. Patients with T3/T4 tumours with/ without neck nodes, ECOG performance status1-2, Age between 45-60 yrs, Informed consent

2. Laboratory investigations criteria were: $\mathrm{Hb}>10 \mathrm{gm} \%$, TLC $>2500$ cells/cc, Platelets $>100000 / \mathrm{cc}$, S. Creatinine $<1.5 \mathrm{mg} / \mathrm{dl}$, S. Bilirubin $<2 \mathrm{mg} / \mathrm{dl}$

31 patients were histopathologically proved anaplastic carcinoma one patient was of medullary carcinoma thyroid.

Treatment Protocol: The patients were divided in to 2 arms of 16 each

Arm I (16) patients received CDDP75 mg $/ \mathrm{m} 2$ (1hr) infusion + doxorubicin $60 \mathrm{mg} / \mathrm{m} 2 \mathrm{I} . \mathrm{V}$.

Arm II (16) patients received CDDP 75/m2 (8 hrs infusion)+ doxorubicin $60 \mathrm{mg} / \mathrm{m} 2 \mathrm{I} / \mathrm{V}$

Hydration with normal saline, mannitol and prophylactic premedication with dexamethasone, diphenhydramine and ondensetron were administered to all patients. LFT, KFT and haematological tests were done before every cycle of chemotherapy.

Patients were observed for toxicities of chemotherapy like nausea and vomiting diarrhea, alopecia, stomatitis ototoxicity, neurotoxicity, cardiotoxicity etc and response was assessed after every cycle of CT.

Response was assessed for primary tumour, nodal disease and distant metastatic sites like lung, bone etc. Response was assessed as: CR- Complete response, PR- Partial response, SD- Stable disease, NR-No response, Pr. DProgressive disease

\section{Follow up:}

First follow up was done after one week of first cycle of chemotherapy to note skin and mucosal toxicities and hematological toxicities. Subsequent follow up at 2 weekly intervals till 1year after completion of treatment, $(6$ cycles of chemotherapy and radiotherapy $60 \mathrm{~Gy}$ ) 
Follow up consisted assessment of response, Toxicities

like haematological, gastrointestinal, nephrotoxicity,
Residual and progressive disease at primary or metastatic site

\section{Results}

Table 1: Response (Locoregional) of Chemotherapy

\begin{tabular}{|c|c|c|}
\hline Response & Arm I (1hr) & Arm \\
& & II (8hr) \\
\hline CR & $2(12.5 \%)$ & $5(31.25 \%)$ \\
\hline PR & $7(43.75 \%)$ & $8(50 \%)$ \\
\hline SD & $3(18.75 \%)$ & $1(6.25 \%)$ \\
\hline Pr. D & $4(25 \%)$ & $2(12.5 \%)$ \\
\hline
\end{tabular}

Arm II (8hr) patients had better loco regional response than Arm I (1hr) in terms of CR /DFS (Disease free survival), PR. $\mathrm{p}=0.02$

Table 2: Response at metastatic sites

Arm I

Arm II

\begin{tabular}{|c|c|c|c|c|c|c|c|c|c|c|c|}
\hline & No & CR & PR & Pr D & SD & & No. & CR & PR & Pr.D & SD \\
\hline Lung & 4 & 0 & $2 / 4(50 \%)$ & $2 / 4(50 \%)$ & - & Lung & 6 & $2 / 6(33 \%)$ & $4 / 6(67 \%)$ & Nil & - \\
\hline Bone & 4 & 0 & $2 / 4(50 \%)$ & $2 / 4(50 \%)$ & & Bone & 4 & $0 \%$ & $4 / 4(100 \%)$ & Nil & - \\
\hline Brain & Nil & & - & - & - & Brain & Nil & & - & - & - \\
\hline
\end{tabular}

Arm II patients showed improved response at metastatic sites like lung $\&$ bones as well, $\mathrm{p}=0.06$

Table 3: Side Effect/ Toxicity: Acute Mucositis

\begin{tabular}{|c|c|c|}
\hline & Arm I & Arm II \\
\hline Grade I & $4(25 \%)$ & $3(18.75 \%)$ \\
\hline Grade II & $7(43.75 \%)$ & $4(25 \%)$ \\
\hline Grade III & $5(31.25 \%)$ & $2(12.5 \%)$ \\
\hline Grade IV & - & - \\
\hline
\end{tabular}

Mucositis Gr II/III was less in Arm II, p=0.08 
Table 4: Side Effect/ Toxicity: Hematological toxicities

Neutropenia

\begin{tabular}{|c|c|c|c|c|}
\hline & Arm I & Arm II & ARM I & ARM II \\
\hline Gr I & $6(37.5 \%)$ & $6(37.5 \%)$ & & $2(12.5 \%)$ \\
\hline Gr II & $6(37.5 \%)$ & $2(12.5 \%)$ & $5(37.5 \%)$ & $6(37.5 \%)$ \\
\hline Gr III & $4(25 \%)$ & - & $6(37.5 \%)$ & $6(37.5 \%)$ \\
\hline Gr IV & - & - & $5(31.25 \%)$ & $2(12.5 \%)$ \\
\hline
\end{tabular}

Myelosuppresion in the form of Anemia and Neutropenia were more common in Arm I, p=0.06

Table 5: Gastrointestinal toxicities / nausea and vomiting:

\section{Diarrhea}

Nausea / Vomiting

\begin{tabular}{|c|c|c|c|c|c|}
\hline & Arm I & Arm II & & ARM I & ARM II \\
\hline Gr I & $2(12.5 \%)$ & $2(12.5 \%)$ & Gr I & $6(37.5 \%)$ & $5(37.5 \%)$ \\
\hline Gr II & $8(50 \%)$ & $3(18.75 \%)$ & Gr II & $6(37.5 \%)$ & $2(12.5 \%)$ \\
\hline Gr III & $6(37.5 \%)$ & $2(12.5 \%)$ & Gr III & $4(25 \%)$ & $1(6.25 \%)$ \\
\hline Gr IV & & & Gr IV & - & - \\
\hline
\end{tabular}

Gastrointestinal toxicities like nausea \& vomiting were significantly less in Arm II p=0.06 and diarrhea was also less common in Arm II, $\mathrm{p}=0.09$

Table 6: Side Effect/ Toxicity: Nephrotoxicity

\begin{tabular}{|c|c|c|}
\hline & ARM I & ARM II \\
\hline G I & $4(25 \%)$ & $1(6.25 \%)$ \\
\hline G II & - & - \\
\hline G III & - & - \\
\hline G IV & - & \\
\hline
\end{tabular}

Nephrotoxicity in the form of rise in Blood urea/ S. creatinine was also more in Arm I than Arm II, which was managed easily with hydration, injection Furesemide and oral Allopurinol.

As sample size was small $\mathrm{p}$ values of $</=0.05$ could not be derived and p values response $\&$ toxicities were between 0.02 0.09 , mean $\mathrm{p}=0.06$ was calculated using chi square test $\&$ graph pad software. 


\section{Discussion}

Anaplastic Thyroid Cancer is one of the most aggressive and difficult human malignancies to treat and subsequently is one of the most lethal. As opposed to the excellent longterm survival for well-differentiated thyroid carcinoma, ATC in most series has a median survival of 4-5 months from the time of diagnosis with rare long-term survivors ${ }^{1,3}$.

Aim of the present study is to evaluate response and toxicities of protracted cisplatinum infusion (8hr) + doxorubicin in comparison to $(1 \mathrm{hr})$ cisplatinum infusion + doxorubicin.

Arm II ( $8 \mathrm{hr}$ ) infusion resulted better locoregional response with CR $31.25 \%$, PR 50\%, SD 6.25\%, Pr.D 12.5\% as compared to Arm 1with CR12.5\%, PR43.75\%, SD $18.75 \%$, Pr.D25\%

Arm II showed improved response in metastatic sites like lung, bones and brain as well with CR $33.3 \%$, PR 50\% in lung, PR $100 \%$ in bone compared to CR $0 \%$, PR $66.6 \%$ in lung and PR 50\% in bone.

Grade IV myelosuppresion is proportionately less in Arm II, Gastrointestinal toxicity which were dose limiting in Arm I were significantly less in Arm II. On follow up in Arm II,5 patients had CR and survived DF 1 year on completion of $\mathrm{CT}+\mathrm{RT}$, whereas there were 2 patients showing CR in Arm I. Eight patients had partial remission lasting 3-4 months in Arm II whereas only 7 patients showed PR lasting 2-3 months in Arm I.

Three patients in Arm I and one patient in Arm II had SD for 3-7 months whereas 4 patients in Arm I died of progressive disease during follow up period of 3-9 months after completion treatment compared to 2 patients who died of Pr.D in Arm II.

Presently very few literatures are available on use of protracted CDDP infusion in anaplastic thyroid cancer. Japanese society of thyroid surgery ${ }^{15}$ evaluated efficacy of prolonged CDDP +doxorubicin + etoposide+ Peplomycin, in 18 patients of Stage III/IV of anaplastic thyroid cancer and 12 patients showed PR for 2-3 months. 3 patients had progressive disease and died in 3-7 months, 3 had stable disease for 3-11 months. Results are comparable to present study. Myelosuppression was the major toxicity observed by them.

Tavecchio et $\mathrm{al}^{19}$ studied trial with prolonged cisplatin infusion through central venous catheter in thoracic malignancies with superior vena cava obstruction.

Jelic S et al $^{12}$ investigated $6 \mathrm{hr}$ cisplatin $+5 \mathrm{FU}$ in 170 Head\& Neck cancer patients with CR 11, SD 15,PR 54, Pr D 20 percentage respectively and overall response $65 \%$, which is to some extent similar to our study.

Arcangeli $G$ et $\mathrm{al}^{18}$ attempted accelerated hyper fractionated RT with protracted concurrent CT with cisplatinum and concluded that this regimen has potential of achieving significant improvement as compared to standard concurrent chemotherapy schedule while avoiding significant toxicities. Grade III or greater systemic toxicity occurred in 9 of 65 (14\%) patients and was never the cause of drug dose reduction.

CR was observed in $69 \%$ of the patients with gross disease and DFS of $45 \%$ was observed in 43.5 months follow up. Response and DFS observed in this study is much higher than our trial.

Hence it can be summarized from the present study which is in accordance with trials of few other researchers that protracted cisplatinum infusion offers better response rates at loco regional and metastatic sites and is much better tolerated especially due to less pronounced gastrointestinal side effects than usual short duration cisplatinum infusion in anaplastic thyroid cancer

\section{Conclusion}

From the present study it can be concluded that protracted cisplatinum (CDDP) infusion combined with other 
chemotherapeutic drugs like doxorubicin is a well-tolerated regime with significantly less systemic toxicity especially (GI toxicity) as compared to $1 \mathrm{hr}$ infusion and can be used effectively as chemotherapy regimen in advanced inoperable anaplastic thyroid and head \& neck cancer.

More studies on protracted cisplatinum in various combinations with bigger sample size in order to reach to a more statistically significant conclusion are required to further establish its efficacy in this variety of cancer.

\section{Funding: Nil}

\section{Conflict of interest: Nil}

\section{Permission from IRB: Yes}

\section{Acknowledgement}

We acknowledge support of our departmental colleagues, staff nurses and the patients who were subject of this study.

\section{References}

1. Nel CJ, Van Herdeen JA, Goellner JR. Anaplastic carcinoma of thyroid: a clinic pathologic study of 82 cases. Mayo clinic Proto. 1985;(60): 51

2. Jossart GH, Clark OH.Well-differentiatedthyroid cancer. Current ProblemsSurg. Dec 1994; 31(12): 9331012

3. Tan RK, Finley RK, Driscoll D. Anaplastic carcinoma of thyroid: a24-year experience.Head\&Neck Oncol. 1995; 17(1): 41-48.

4. AinKB, Egorin MJ, DesimonePA. Treatment of anaplastic thyroid carcinoma with Paclitaxel: Phase II trial using ninety-six hour infusion, Collaborative Anaplastic thyroid Cancer Health Intervention Trials(CATCHIT) group.Thyroid. July2000; 10(7): 587-594

5. Parkin DM, FerlayJ, Bray F, PisaniP. Estimating world cancer burden: GLOBOCAN 2000. Int. J. of cancer. Oct 2001; 94(2): 153-156
6. Vincent Devita, SamuelHellman, Steven A. Rosenberg.Cancer: PrinciplesandPracticeofOncology; $6^{\text {th }}$ Ed: Lippincott Williams \& Willkins.

7. Schlumberger MJ, Anaplastic thyroid carcinoma. Orpha-net encyclopedia, March 2004; 1-3

8. Mitchell G, Huddart R, Harmer C. Phase II evaluation of high dose accelerated radiotherapy for anaplastic thyroid carcinoma.RadiotherapyOncol.Jan1999; 50 (1): 33-38.

9. Kim JH, Leeper RD. Treatment of anaplastic giant and spindle carcinoma of the thyroid glandwith combination of Adriamycin and radiotherapy: a new approach, Cancer, $1983 ; 52: 954$

10. Hundahla SA, Fleming ID, Fremgen AM. A National Cancer Database report on 856 case of thyroid carcinoma treated in U.S: 1985-1995. Cancer.1998; 83(12): 2638.

11. Shimaoka K. A randomized trial of doxorubicin versus doxorubicin plus Cisplatin in patients with advanced thyroid cancer.Cancer.1985; (56): 2155-60.

12. Jelic S, StamaovicLJ, Vucicevic S. Use of high dose cytarabine to enhance Cisplatin cytotoxicity effects on the response and overall survival rates of advanced head \&neck cancer patients .Eur J of Cancer. 2002Jul; 38(11): 1478-89.

13.Creagan ET, FallonO, Woods JE. CDDP administered by $24 \mathrm{hr}$ infusion in treatment of patients with advanced upper aerodigestive cancer.Cancer.1983 Jun: 51(11): 2020 3.

14. BusnardoB, DanielO, PelizzoMR . A multimodality therapeutic approach in anaplastic thyroid cancer: Study on 39 patients. J Endocrinol Invest .2000 Dec; 23(11): 755-61

15. Chemotherapy Committee, The Japanese Society of thyroid Surgery. Intensive chemotherapy for anaplastic thyroid carcinoma: Combination of Cisplatin,

Doxorubicin, etoposide and peplomycin with granulocyte colony stimulating factor support. Jpn J Clin Oncol.1995Oct; 25(5): 203-7. 
16. Voigt W, BulankinA, Muller T. Schedule dependent antagonism of Gemcitabine and Cisplatin in human anaplastic thyroid cancer cell lines. Clin Cancer Res. 2000 May; 6(5): 2087-93.

17. West DC, Shamberger RC, Macklis RM. Stage III neuroblastoma over 1 year of age at diagnosis, improved survival with intensive multimodality therapy including multiple alkylating agents. J. Clin Oncol; 1993 (11): 84-90.
18. Arcangeli G, Saracino B, Danesi DT. Accelerated hyperfractionated radiotherapy and concurrent protracted venous infusionchemotherapy in locally advanced Head \& neck cancer, Am J Clin Oncol. Oct 2002;(5): 431-7.

19. Tavecchio L, BediniAV, LanocitaR.Long term infusion in cancer chemotherapy with the Groshong catheter via the inferior vena cava. Tumori. 1996 Jul-Aug; 82(4): 372-5

\section{How to cite this article?}

Ghosh G, Singh OP. Evaluation of Protracted Cisplatinum Infusion in Advanced Anaplastic Thyroid Cancer. Int J Med Res Rev 2013;1(4):162-168. doi:10.17511/ijmrr.2013.i04.04 